\title{
Exact-exchange-based quasiparticle energy calculations for the band gap, effective masses, and deformation potentials of $\mathrm{ScN}$
}

\author{
Abdallah Qteish \\ Department of Physics, Yarmouk University, 21163-Irbid, Jordan \\ Patrick Rinke and Matthias Scheffler \\ Fritz-Haber-Institut der Max-Planck-Gesellschaft, Faradayweg 4-6, D-14195 Berlin-Dahlem, Germany
}

Jörg Neugebauer

MPI für Eisenforschung, Max-Planck Straße 1, D-40237 Düsseldorf, Germany

(Received 19 August 2006; published 13 December 2006)

\begin{abstract}
The band gaps, longitudinal and transverse effective masses, and deformation potentials of $\mathrm{ScN}$ in the rock-salt structure have been calculated employing $G_{0} W_{0}$-quasiparticle calculations using exact-exchange Kohn-Sham density-functional theory one-particle wave functions and energies as input. Our quasiparticle gaps support recent experimental observations that $\mathrm{ScN}$ has a much lower indirect band gap than previously thought. The results are analyzed in terms of the influence of different approximations for exchange and correlation taken in the computational approach on the electronic structure of $\mathrm{ScN}$.
\end{abstract}

DOI: 10.1103/PhysRevB.74.245208

PACS number(s): 71.20.Nr, 71.15.Mb

\section{INTRODUCTION}

Scandium nitride $(\mathrm{ScN})$ is emerging as a versatile material for promising technological applications. As part of the transition-metal nitride family it initially generated interest for potential applications as wear-resistant and optical coatings due to its mechanical strength, high melting point of $2600{ }^{\circ} \mathrm{C},{ }^{1}$ and high hardness $(H=21 \mathrm{GPa})$ with respect to load deformations. ${ }^{2} \mathrm{ScN}$ crystallizes in the rock-salt phase with a lattice parameter of $4.50 \AA .{ }^{3}$ The octahedral bonding arrangement provides a much more favorable environment for the incorporation of transition-metal atoms like $\mathrm{Mn}$ or $\mathrm{Cr}$ than the tetrahedrally coordinated III-V semiconductors, which have up until now been popular candidates for spintronic materials. Successful incorporation of $\mathrm{Mn}$ into $\mathrm{ScN}$ has been demonstrated ${ }^{4}$ and $a b$ initio calculations predict Mn-doped $\mathrm{ScN}$ to be a dilute ferromagnetic semiconductor. ${ }^{5}$ Moreover, ScN has a lattice mismatch of less than $2 \%$ to cubic gallium nitride $(\mathrm{GaN})$. This makes $\mathrm{ScN}$ structurally compatible with the group-IIIA nitrides ${ }^{6-13}$ —an important technological material class, in particular, for applications in optoelectronic devices. Alloying $\mathrm{ScN}$ with GaN (Refs. 9-12) might provide a viable alternative to InGaN alloys for use in light-emitting devices or solar cells. In addition, multifunctional devices are conceivable if the strong electromechanical response predicted for hexagonal ScN (Ref. 14) can be utilized.

The electronic band structure of $\mathrm{ScN}$ - a key quantity for the design of optoelectronic devices-has been difficult to access both experimentally and theoretically. Early experiments were hampered by various complications in growing films with well-defined crystalline orientation, stoichiometry, low background carrier concentration, and surface roughness. For a detailed discussion we refer to, e.g., Ref. 15. Recent advances in growth techniques have led to a systematic improvement of the material's quality ${ }^{16}$ Employing optical spectroscopy and photoemission, Gall et al. ${ }^{15}$ concluded that $\mathrm{ScN}$ is a semiconductor with an indirect $\Gamma-X$ band gap $\left(E_{g}^{\Gamma-X}\right)$ of $1.3 \pm 0.3 \mathrm{eV}$. The sizable error bar of $0.3 \mathrm{eV}$ has been mainly attributed to the large background carrier concentration of $\sim 5 \times 10^{20} \mathrm{~cm}^{-3}$ causing an apparent increase of the band gap due to the Burnstein-Moss shift. ${ }^{17}$ Reducing the electron carrier concentration to $4.8 \times 10^{18} \mathrm{~cm}^{-3}$ and combining tunneling spectroscopy and optical-absorption measurements, Al-Brithen et al. ${ }^{18}$ were able to reduce the error bar and found a value for $E_{g}^{\Gamma-X}$ of $0.9 \pm 0.1 \mathrm{eV}$.

Early Kohn-Sham density-functional theory (KS-DFT) calculations employing the local-density (LDA) or $X \alpha$ approximations predicted $\mathrm{ScN}$ to be a semimetal with a small negative band gap between -0.01 and $-0.21 \mathrm{eV} .{ }^{19-21}$ In order to overcome the well-known underestimation of the LDA band gap, more advanced exact-exchange [OEPx(cLDA)] (Ref. 15) and screened-exchange ${ }^{22}$ calculations have been performed, and showed that $\mathrm{ScN}$ is a semiconductor with an indirect $\Gamma$ to $X$ band gap, in accord with experimental evidence. ${ }^{15,18}$ However, the calculated band gap of $1.60 \mathrm{eV}$ found in both studies is significantly larger than the most recent experimental value of $0.9 \pm 0.1 \mathrm{eV} .{ }^{18}$

In order to shed light on this discrepancy we have performed quasiparticle energy calculations in Hedin's $G W$ approximation, ${ }^{23}$ which is a well-established technique to calculate accurate band-structure energies and currently the choice for computing quasiparticle band structures of solids. ${ }^{24-26}$ The quasiparticle calculations predict $\mathrm{ScN}$ in the rock-salt phase to have an indirect band gap between the $\Gamma$ and $X$ point of $0.99 \pm 0.15 \mathrm{eV}$, strongly supporting recent experimental findings. In addition, we have also determined the direct band gaps and other electronic structure parameters relevant for device simulations: the volume deformation potentials of the main band gaps and the longitudinal and transverse effective masses of the conduction band at the $X$ point. The effective mass has previously been calculated at the level of the LDA, ${ }^{27}$ but to the best of our knowledge, only one experimental study has reported a conduction-band ef- 
fective mass for $\mathrm{ScN}$ (between 0.1 and $\left.0.2 m_{0}\right)^{28}$ so far. For the deformation potentials no experimental or theoretical data is available, yet.

Most commonly, the Greens function $G_{0}$ and the screened potential $W_{0}$ required in the $G W$ approach (henceforth denoted $G_{0} W_{0}$ ) are calculated from a set of KS-DFT singleparticle-energies and wave functions $\left\{\epsilon_{i}, \phi_{i}\right\}$. Since $G_{0}$ and $W_{0}$ are not usually updated by the quasiparticle wave functions and energies $\left\{\epsilon_{i}^{Q P}, \phi_{i}^{Q P}\right\}$ in a self-consistent manner, the quasiparticle energies depend on the approximation used to calculate the input data. ${ }^{25,29-31}$

Originally, $G_{0} W_{0}$ calculations were based on LDA data (LDA- $G_{0} W_{0}$ ) and were found to accurately predict band gaps of $s p$-bonded semiconductors (with a typical error bar of $\sim 0.1 \mathrm{eV}) .{ }^{24}$ However, complications arise when the LDA$G_{0} W_{0}$ approach is used to calculate the electronic structure of semiconductors with negative LDA band gaps ${ }^{32,33}$ or when occupied shallow semicore $d$ bands are treated as valence in the pseudopotential framework. ${ }^{34-36}$ For such semiconductors, $G_{0} W_{0}$ calculations based on OEPx(cLDA) data $\left[\mathrm{OEPx}(\mathrm{cLDA})-G_{0} W_{0}\right]$ have been found to provide a reliable tool to obtain band gaps with an accuracy of $0.1 \mathrm{eV}^{25,29}$

The key to the improved description in the OEPx(cLDA)$G_{0} W_{0}$ approach can be found in the treatment of exchange. In the exact-exchange KS approach the formal expression for the total energy is the same as in Hartree-Fock. The difference between the two methods lies in the potential felt by the electrons: in Hartree-Fock the exchange potential is nonlocal, whereas in the exact-exchange KS approach it is local and constructed to be the variationally best local groundstate potential to the nonlocal Hartree-Fock exchange potential. ${ }^{37}$ Like Hartree-Fock, the exact-exchange KS approach is therefore free of self-interaction, but since the eigenvalues are solutions to a local potential they are, in general, closer to (inverse) photoemission data for semiconductors than the Hartree-Fock single-particle energies. ${ }^{38-40}$ The $G W$ formalism, on the other hand, goes beyond the KS approach and describes the interaction of weakly correlated quasiparticles by means of a nonlocal, energy-dependent self-energy. It takes the form of the nonlocal exchange potential encountered in the Hartree-Fock approach, which is screened by correlation in the random-phase approximation (RPA). To elucidate the effects of exchange and correlation on the electronic structure of $\mathrm{ScN}$ we therefore first analyze the influence of exchange by comparing LDA (GGA) and OEPx (cLDA) calculations, before turning to the difference between OEPx(cLDA) and $G_{0} W_{0}$.

The paper is organized as follows. Section II describes our computational approach. The results are presented and discussed in Sec. III. Finally, a summary is given in Sec. IV.

\section{COMPUTATIONAL METHOD}

The KS-DFT calculations have been performed with the $a b$ initio pseudopotential (PP) plane-wave code SPHInX. ${ }^{41} \mathrm{~A}$ consistent set of norm-conserving scalar-relativistic PPs has been used for each of the exchange-correlation functionals [LDA, GGA, and OEPx(cLDA)]. The OEPx(cLDA)-PPs have been constructed as described in Ref. 42 and the LDA and GGA ones using the FHI98PP code. ${ }^{43}$ All PPs for both Sc and $\mathrm{N}$ have been generated according to the TroullierMartins optimization scheme ${ }^{44}$ and have then been transformed into the separable Kleinman-Bylander form. ${ }^{45}$ Unlike in the group-IIIA nitrides $\mathrm{GaN}$ and $\mathrm{InN}$ the cation $3 s$ and $3 p$ states have moderate binding energies in $\mathrm{ScN}$ and the $\mathrm{Sc} 3 p$ and to a lesser degree also the Sc $3 s$ electrons couple to the upper valence bands as a partial charge-density analysis reveals (see Sec. III A and also Fig. 4 in Ref. 22). For Sc, the entire semicore shell ( $3 s, 3 p$, and $3 d$ states) has therefore been treated as valence with an ionic configuration $[\mathrm{Ne}] 3 s^{2} 3 p^{6} 3 d^{1}$. Following Ref. 46, only the $2 s$ and $2 p$ components have been included for $\mathrm{N}$. Adding a $d$ component for $\mathrm{N}$ yields negligible effects on the calculated band structure and total energies of $\mathrm{ScN}$. For $\mathrm{Sc}$, we have chosen a core radius of $1.4,1.4$, and 1.8 bohrs for the $s, p$, and $d$ orbitals, respectively. For N, a common core radius of 1.5 bohrs has been adopted. The $s(p)$ component is taken as the local component for $\mathrm{Sc}(\mathrm{N})$. Only one projector per angular momentum channel has been used, i.e., $3 p$ and $3 d$ for Sc. We have verified that this procedure does not compromise higher-lying states in the same channel by ensuring that the eigenvalues of the $4 s$ and $4 p$ levels in the pseudoatom reproduce those of the all-electron calculation. These pseudopotentials have been carefully tested (see also below) and are free of ghost states. ${ }^{47}$

For the LDA calculations we have used the Ceperley-Alder ${ }^{48}$ exchange-correlation data as parametrized by Perdew and Zunger. ${ }^{49}$ The GGA calculations have been performed with the Perdew-Burke-Ernzerhof ${ }^{50}$ functional. In the OEPx(cLDA) calculations, the exchange energy and potential have been treated exactly and correlation has been added on the LDA level. Throughout the paper, the combination of exact-exchange and LDA correlation will be referred to as OEPx(cLDA).

The $G_{0} W_{0}$ calculations have been performed employing the $G W$ space-time approach, ${ }^{51}$ in the GWST implementation. $^{52-54}$ The Kohn-Sham eigenvalues and wave functions $\left(\epsilon_{i}\right.$ and $\left.\phi_{i}\right)$ in either OEPx(cLDA) or LDA are used as input to construct $G_{0}$ and $W_{0}$. Head and wings of the dielectric matrix (which converge slowly with respect to the $\mathbf{k}$ mesh) have been calculated using a fine $10 \times 10 \times 10$ MonkhorstPack (MP) mesh. ${ }^{55}$ We find that using an offset of $\left[\frac{1}{2}, \frac{1}{2}, \frac{1}{2}\right]$ yields faster convergence with respect to the number of $k$ points, because the $k$-point set then contains fewer highsymmetry points. Contributions arising from the nonlocal part of the pseudopotential are fully taken into account. ${ }^{54} \mathrm{~A}$ regular $4 \times 4 \times 4$ MP mesh centered on the $\Gamma$ point then proves to be sufficient for the full $G_{0} W_{0}$ calculations.

Brillouin-zone integrations in the DFT calculations have been performed on a $4 \times 4 \times 4$ MP mesh. In all calculations an energy cutoff of $80 \mathrm{Ry}$ is used for the plane-wave expansion of the wave functions. For the independent-particle polarizability $\chi_{0}$, which enters in the calculation of the OEPx(cLDA) potential (see, e.g., Ref. 39), an energy cutoff of $55 \mathrm{Ry}$ gives converged results. Conduction-band states up to the same energy cutoff have been included in the calculation of the electronic Green's function in the OEPx(cLDA) as well as in the $G W$ calculations. These parameters yield converged KS and quasiparticle energies to within $0.05 \mathrm{eV}$. 
TABLE I. Structural parameters of ScN: lattice constant $\left(a_{0}\right)$, bulk modulus $\left(B_{0}\right)$, and its derivative $\left(B_{0}^{\prime}\right)$ calculated in our pseudopotential, plane-wave (PP-PW) approach compared to previous results obtained with the all-electron full-potential linearized augmented plane-wave approach (FP-LAPW) and experiment.

\begin{tabular}{lccc}
\hline \hline \multicolumn{1}{c}{$\begin{array}{c}\text { Approach } \\
\text { (Present work) }\end{array}$} & $a_{0}(\AA)$ & $B_{0}(\mathrm{GPa})$ & $B_{0}^{\prime}$ \\
\hline PP-PW(LDA) & 4.455 & 221 & 4.27 \\
PP-PW(GGA) & 4.533 & 196 & 4.36 \\
\multicolumn{4}{c}{ Other theoretical calculations } \\
\hline FP-LAPW(LDA) (Ref. 22) & 4.42 & 235 \\
FP-LAPW(LDA) (Ref. 71) & 4.44 & 220 & \\
FP-LAPW(GGA) (Ref. 22) & 4.50 & 201 & \\
FP-LAPW(GGA) (Ref. 71) & 4.54 & 201 & 3.31 \\
Experiment (Ref. 2) & 4.501 & $182 \pm 40$ \\
\hline \hline
\end{tabular}

We have tested the reliability of our pseudopotential calculations against all-electron calculations in the full-potential linearized augmented plane-wave (FP-LAPW) approach, where available. For ground-state calculations on the level of LDA and GGA we find good agreement for the structural properties, as Table I illustrates. LDA slightly underestimates the lattice constant of $\mathrm{ScN}$ compared to experiment ${ }^{2}$ by approximately $1 \%$, whereas GGA results in a slight overestimation.

The issue of performing self-consistent $G W$ calculations is still a matter of debate. ${ }^{56-60}$ Unlike in DFT, a selfconsistent solution of the full set of equations for the selfenergy in many-body perturbation theory would go beyond the $G W$ approximation and successively introduce higherorder electron-electron interactions with every iteration step. Solving the $G W$ equations self-consistently is therefore inconsistent if no higher-order electron-electron interactions are included. It was first observed for the homogeneous electron gas ${ }^{61}$ that the spectral features broaden with an increasing number of iterations in the self-consistency cycle. Similarly, for closed-shell atoms the good agreement with experiment for the ionization energy after the first iteration is lost upon iterating the equations to self-consistency. ${ }^{58}$ Imposing self-consistency in an approximate fashion ${ }^{36,60,62,63}$ is not unique and different methods yield different results. Since the issue of self-consistency within $G W$ is still discussed controversially, we refrain from any self-consistent treatment and remain with the zeroth order in the self-energy $\left(G_{0} W_{0}\right)$. We argue (see Sec. III A) that in the case of $\mathrm{ScN}$ the error bar resulting from this approximation is only of the order of $0.15 \mathrm{eV}$.

\section{RESULTS AND DISCUSSION}

\section{A. Electronic band structure}

The quasiparticle band structure of $\mathrm{ScN}$ is calculated employing both the OEPx(cLDA)- $G_{0} W_{0}$ and LDA- $G_{0} W_{0}$ approach. To understand the effect of the starting point on the $G_{0} W_{0}$ calculations we first analyze the KS band structure

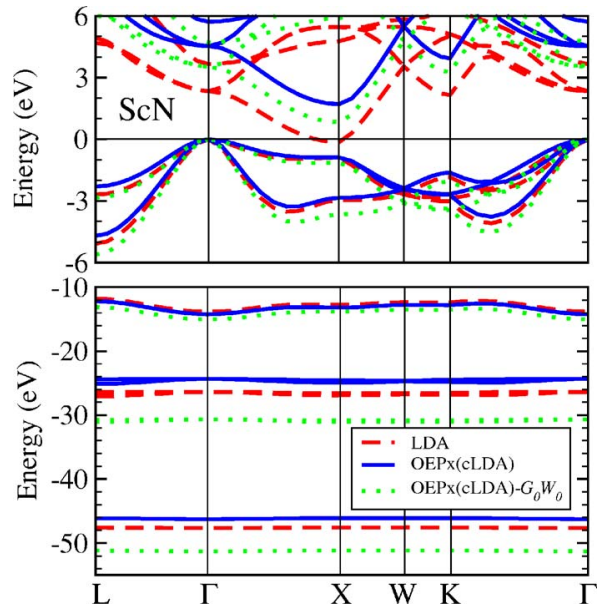

FIG. 1. (Color online) Electronic band structure of rock-salt $\mathrm{ScN}$ in LDA, OEPx(cLDA), and $\operatorname{OEPx}(\mathrm{cLDA})-G_{0} W_{0}$. The top panel shows the upper valence and lower conduction bands, aligned at the top of the valence band. The bands in the lower panel have mainly $\mathrm{N} 2 s$ (around $-13 \mathrm{eV}$ ), Sc $3 p$ (around $-30 \mathrm{eV}$ ), and Sc $3 s$ character (around $-50 \mathrm{eV}$ ).

using three levels of approximations for the $X C$ potential [OEPx(cLDA), GGA, and LDA]. For a meaningful comparison between the results of these calculations among themselves and with experiment, these electronic-structure calculations have been performed at the experimental equilibrium volume. As an example, we show in Fig. 1 the electronic band structures of $\mathrm{ScN}$ in LDA, OEPx(cLDA) and $\operatorname{OEPx}(\mathrm{cLDA})-G_{0} W_{0}$. Table II summarizes the calculated band gaps $\left(E_{g}^{\Gamma-X}, E_{g}^{X-X}\right.$, and $\left.E_{g}^{\Gamma-\Gamma}\right)$, previous $\operatorname{OEPx}(\mathrm{cLDA})^{15}$ and screened-exchange ${ }^{22}$ results and experimental data. ${ }^{15,18}$ For the following discussion we consider only the latest experimental data of Al-Brithen et al. on low background carrier samples ${ }^{18}$ as a reference.

TABLE II. Calculated and experimental band gaps $\left(E_{g}\right)$ of $\mathrm{ScN}$ (in $\mathrm{eV}$ ). sX denotes previous screened exchange calculations and $\left[G_{0} W_{0}\right]_{\text {average }}$ the arithmetic average between the OEPx(cLDA)$G_{0} W_{0}$ and LDA- $G_{0} W_{0}$ results (see text).

\begin{tabular}{lccc}
\hline \hline \multicolumn{1}{c}{$\begin{array}{c}\text { Approach } \\
\text { (Present work) }\end{array}$} & $E_{g}^{\Gamma-\Gamma}$ & $E_{g}^{\Gamma-X}$ & $E_{g}^{X-X}$ \\
\hline OEPx(cLDA)- $G_{0} W_{0}$ & 3.51 & 0.84 & 1.98 \\
LDA- $G_{0} W_{0}$ & 3.71 & 1.14 & 2.06 \\
{$\left[G_{0} W_{0}\right]_{\text {average }}$} & 3.62 & 0.99 & 2.02 \\
OEPx(cLDA) & 4.53 & 1.70 & 2.59 \\
GGA & 2.43 & -0.03 & 0.87 \\
LDA & 2.34 & -0.15 & 0.75 \\
Other theoretical work & & & \\
\hline OEPx(cLDA) (Ref. 15) & 4.70 & 1.60 & 2.90 \\
sX (Ref. 22) & & 1.58 & 2.41 \\
Experiment & & & \\
\hline Ref. 15 & $\sim 3.8$ & $1.30 \pm 0.3$ & 2.40 \\
Ref. 18 & & $0.9 \pm 0.1$ & 2.15 \\
\hline \hline
\end{tabular}




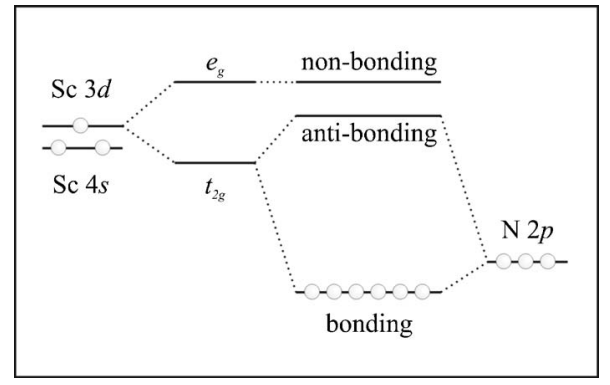

FIG. 2. Schematic diagram of the $p d$ bonding in rock-salt $\mathrm{ScN}$ : the Sc $3 d$ states are split into $t_{2 g}$ and $e_{g}$ states by the crystal field. The $t_{2 g}$ states then form bonding and antibonding bands with the $\mathrm{N}$ $2 p$ states and the $e_{g}$ states nonbonding bands that lie higher in energy. The occupation of the relevant states and bands is shown by circles.

In $\mathrm{ScN}$ the scandium atom donates its two $4 s$ and single $3 d$ electron to the nitrogen atom. According to the bonding analysis of Harrison and Straub, ${ }^{64}$ the five $d$ states of Sc hybridize with the three-valence $p$ states of the neighboring $\mathrm{N}$ atoms in the rock-salt structure of $\mathrm{ScN}$, forming three $p$-like bonding, three $d$-like antibonding $t_{2 g}$, and two $d$-like nonbonding $e_{g}$ bands. The bonding scheme together with the electron filling of these bands is sketched in Fig. 2. Performing a partial charge-density analysis we have confirmed that the upper three valence bands in the DFT calculations correspond to the bonding states and originate mainly from the $\mathrm{N}$ $2 p$ states with some admixture of the Sc $3 d$ states, while the lowest conduction bands are the antibonding $t_{2 g}$ states with Sc $3 d$ character. The two bands derived from the nonbonding $e_{g}$ states are around $1.2 \mathrm{eV}$ higher in energy. This assignment is consistent with the partial density-of-states analysis of Stampfl et al. ${ }^{22}$ The character of the deeper-lying bands is given in the caption of Fig. 1.

Focusing first on the band gaps presented in Table II we note that both LDA and GGA underestimate the fundamental indirect by more than $100 \%$. The GGA band gaps are only marginally $(\sim 0.1 \mathrm{eV})$ larger than those of LDA. The reason is a combination of three factors: (1) in direct and inverse photoemission experiments electron addition and removal energies are probed, but the derivative discontinuity of the exchange-correlation potential with respect to changes in the particle number is not taken into account in KS-DFT singleparticle energy calculations, ${ }^{65}$ (2) LDA and GGA are approximate exchange-correlation functionals, which (3) suffer from inherent self-interaction effects. The OEPx(cLDA) formalism also does not fulfill criterion (2), but it is selfinteraction free. This leads to a significant opening of the Kohn-Sham band gaps compared to those of LDA and GGA (Refs. 38-40) as is evident from Table II. Although the OEPx(cLDA) formalism exhibits a derivative discontinuity ${ }^{66,67}$ and therefore fulfills criterion (1) this is of no benefit in KS-DFT single-particle energy calculations. When the excitation energies are calculated by computing total-energy differences in OEPx(cLDA) between the $N$ and the $N \pm 1$ electron system [frequently denoted $\Delta$ selfconsistent field $(\triangle \mathrm{SCF})$ approach], the derivative discontinuity is taken into account properly. ${ }^{66}$ In KS-DFT, however, the

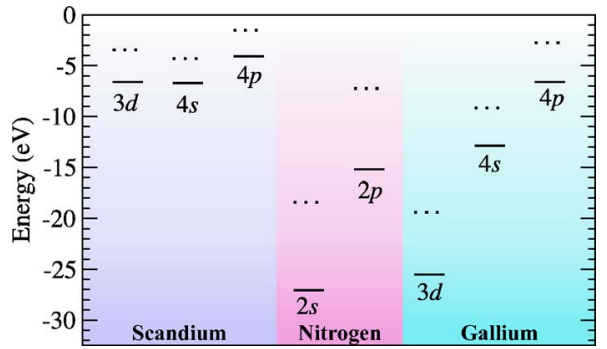

FIG. 3. (Color online) Eigenvalue spectrum of the isolated Sc atom compared to $\mathrm{N}$ and Ga. Dotted lines show the levels in the LDA and solid lines in OEPx(cLDA).

excitation energies are approximated by Kohn-Sham eigenvalue differences of the $N$-electron system alone. The derivative discontinuity hence does not enter the calculation and all states experience the same exchange-correlation potential.

Having established that the removal of the self-interaction in the OEPx(cLDA)-KS approach is the distinguishing feature compared to KS-LDA or KS-GGA calculations we will now illustrate how this leads to an opening of the band gap in $\mathrm{ScN}$. For this it is illuminating to start from the eigenvalues of the isolated $\mathrm{Sc}$ and $\mathrm{N}$ atoms, depicted in Fig. 3. The removal of the self-interaction in OEPx(cLDA) leads to a downward shift of all atomic states. Since the electrons in the second shell of the nitrogen atom are more localized than the electrons populating the third and fourth shells in scandium the self-interaction correction to the $\mathrm{N} 2 p$ state is much larger than that of the Sc $3 d$ state. Inspection of the difference between the exchange potential in OEPx(cLDA) $\left(v_{x}^{\mathrm{OEPx}(\mathrm{cLDA})}\right)$ and LDA $\left(v_{x}^{\mathrm{LDA}}\right)$ shown in Fig. 4(a) reveals that the large relative shift of the atomic N $2 p$ state also translates to the solid. Figure 4(a) illustrates that $v_{x}^{\mathrm{OEPx}(\mathrm{cLDA})}$ is significantly higher than $v_{x}^{\mathrm{LDA}}$ in the Sc regions and lower around the $\mathrm{N}$ atoms. This difference in $v_{x}$ leads to a significant charge-density redistribution [shown in Fig. 4(b)]. The charge transfer from the $\mathrm{Sc}$ to the $\mathrm{N}$ regions gives rise to an increase in the bond ionicity, which, in turn, leads to an opening of the band gap-consistent with our OEPx(cLDA) band-structure calculations.

In the II-VI compounds and group-III nitrides this mechanism is also responsible for an opening of the band gap in OEPx(cLDA) compared to LDA, but it is complemented by a contribution arising from the coupling between the anion semicore $d$ electrons and the $2 p$ electrons of nitrogen. Taking $\mathrm{GaN}$ as an example again, the $\mathrm{Ga} 3 d$ electrons of gallium are energetically lower than the $2 p$ electrons of nitrogen, while in Scandium the Sc $3 d$ lie above the N $2 p$ states (cf Fig. 3). Not the anion $3 d$, like in $\mathrm{ScN}$, but the $\mathrm{Ga} 4 s$ electrons thus form the lower conduction bands with the nitrogen $2 p$ states. Since the OEPx(cLDA) shift of the N $2 p$ states is larger than that of the Ga $4 s$ states the bond ionicity and hence the band gap increase just like in $\mathrm{ScN}$. In addition, the Ga $3 d$ electrons localize stronger in $\mathrm{GaN}$ when the self-interaction is removed in the OEPx(cLDA) approach. ${ }^{25}$ As a result the $p d$ repulsion reduces and the valence bands are lowered in energy leading to a further opening of the band gap. ${ }^{68}$

We now turn to the quasiparticle band structure. In the $\operatorname{OEPx}(\mathrm{cLDA})-G_{0} W_{0}$ approach the band structure is calcu- 


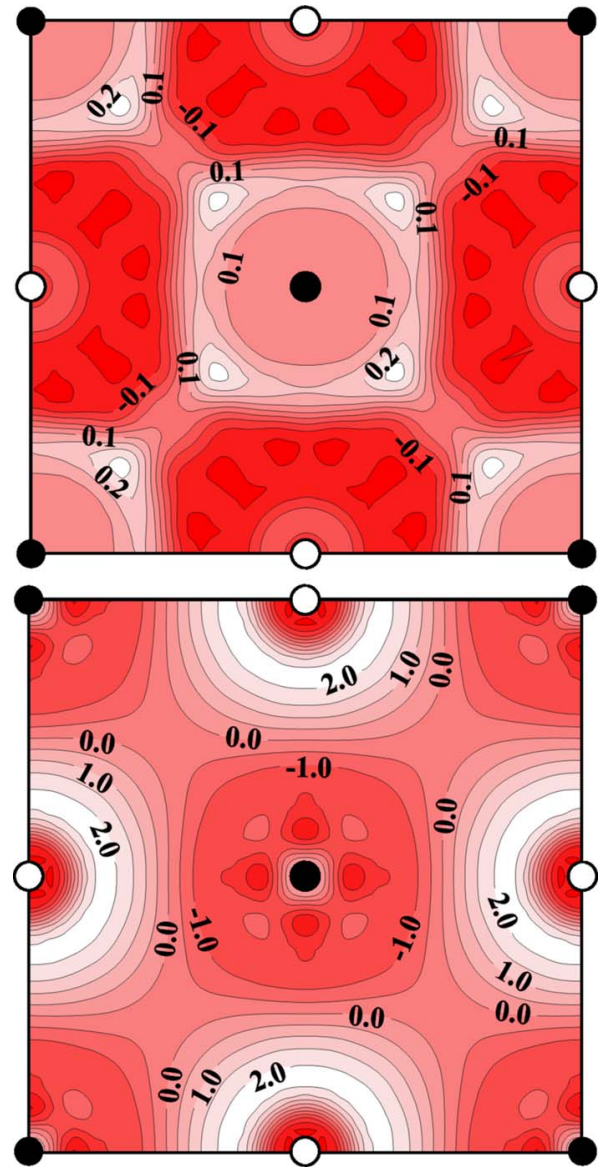

FIG. 4. (Color online) The difference between (a) the OEPx(cLDA) and LDA exchange potentials (in Hartree) and (b) the electronic charge densities (in electrons/unit cell) of $\mathrm{ScN}$ for one of the square faces of the conventional rock-salt unit cell. Black circles denote $\mathrm{Sc}$ and white circles denote $\mathrm{N}$ atoms.

lated directly at the experimental lattice constant. However, the negative LDA band gap (see Table II) impedes the application of this direct approach in the LDA- $G_{0} W_{0}$ formalism with the GWST code, since in its current implementation ${ }^{52,53}$ a clear separation between conduction and valence bands is required. Therefore, an indirect approach is adopted. First, LDA- $G_{0} W_{0}$ calculations are performed at a lattice constant $\left(a_{0}=4.75 \AA\right)$ larger than the experimental one, where the fundamental band gap in the LDA is small but positive. We then use the LDA volume deformation potentials (see Sec. III B) to determine the corresponding LDA- $G_{0} W_{0}$ band gaps at the equilibrium lattice constant. Using the volumedeformation potentials of the LDA instead of the quasiparticle ones is a well-justified approximation, as we will show in the next subsection. While this approach is, in principle, not limited to band gaps, it proves to be too cumbersome for a whole band-structure calculation, because for every bandstructure point the corresponding deformation potential would have to be determined.

The quasiparticle band structure is shown in Fig. 1 and the direct band gaps at the $\Gamma$ and $X$ point are presented together with the indirect gap between $\Gamma$ and $X$ in Table II. It is interesting to note that the LDA- $G_{0} W_{0}$ and OEPx(cLDA)-
$G_{0} W_{0}$ calculations, starting from the two extremes [negative band gap in LDA, $0.8 \mathrm{eV}$ overestimation in OEPx(cLDA)], yield quasiparticle band gaps that agree to within $0.3 \mathrm{eV}$. Since the LDA-based calculations are close to the limit of metallic screening, whereas the OEPx(cLDA)-based calculations form the opposite extreme of starting from a completely self-interaction free exchange-correlation functional, we expect the results of a self-consistent $G W$ calculation to fall in the range between the LDA- $G_{0} W_{0}$ and OEPx(cLDA)$G_{0} W_{0}$ calculations. From these results we estimate the error bar associated with omitting self-consistency in $G W$ to be of the order of $0.15 \mathrm{eV}$ for $\mathrm{ScN}$. Taking OEPx(cLDA) $-G_{0} W_{0}$ results as lower and those of the LDA- $G_{0} W_{0}$ as upper bounds, the arithmetic averages for $E_{g}^{\Gamma-X}, E_{g}^{\Gamma-\Gamma}$, and $E_{g}^{X-X}$ are $0.99,3.62$, and $2.02 \mathrm{eV}$, respectively. These are significantly lower than those from OEPx(cLDA) and the more approximate screened-exchange calculations, as Table II demonstrates. Our quasiparticle gaps clearly support recent experimental findings of an indirect gap of $0.9 \pm 0.1 \mathrm{eV}$ (Ref. 18) and are at the lower bound of earlier measurements on samples with unintentionally high background carrier concentration $(1.3 \pm 0.3 \mathrm{eV}) .^{15}$

The fact that LDA- $G_{0} W_{0}$ and OEPx(cLDA) $G_{0} W_{0}$ calculations yield very similar quasiparticle band gaps is in disagreement with our previous observation for II-VI compounds and GaN. ${ }^{25}$ The difference between $\mathrm{ScN}$ and these compounds is that for the latter the cation semicore $d$ shell is fully filled and the remaining $s$ and $p$ electrons in the semicore shell are much lower in energy. Taking GaN as an example the $3 s$ electrons in the Gallium atom are approximately $100 \mathrm{eV}$ and the $3 p$ electrons approximately $60 \mathrm{eV}$ lower than in scandium. Unlike in $\mathrm{ScN}$ the $3 p$ derived bands therefore show no noticeable dispersion in GaN (cf. Fig. 1). Resolving these more strongly localized $3 s$ and $3 p$ electrons in $\mathrm{GaN}$ with plane waves will thus require significantly higher plane wave cutoffs ${ }^{25}$ than the 80 Ry used in the present study for $\mathrm{ScN}$. In a pseudopotential framework it would hence make sense to explicitly include the $d$ electrons of the cations in the II-VI compounds and group-III nitrides as valence electrons, but to freeze the chemically inert semicore $s$ and $p$ electrons in the core of the pseudopotential. However, due to the large spatial overlap of the atomic semicore $s$ and $p$ with the $d$ wave functions, core-valence exchange is large in these compounds. As a consequence corevalence exchange is treated inconsistently when going from LDA to LDA- $G_{0} W_{0}$, if pseudopotentials are used in this fashion, because the exchange self-energy in the $G W$ approach acts on the $d$ electrons in the solid, but cannot act on the $s$ and $p$ electrons in the semicore shell, too. The result is a severe underestimation of the LDA- $G_{0} W_{0}$ band gaps and $d$ bands that are pushed energetically into the $p$-derived valence bands in the II-VI compounds. ${ }^{25,34,35}$

The only way to remedy this problem within LDA- $G_{0} W_{0}$ is to free the electrons in question by performing all-electron $G_{0} W_{0}$ calculations $^{32}$ or by using pseudopotentials that include the entire shell as valence electrons, ${ }^{34-36}$ which in the latter case introduces formidably high plane-wave cutoffs. If, on the other hand, OEPx(cLDA) is used for the ground-state calculation, then the exchange self-energy already acts on the semicore $s$ and $p$ states in the generation of the pseudopo- 
TABLE III. Transverse $\left(m_{t}^{*}\right)$ and longitudinal $\left(m_{l}^{*}\right)$ effective masses of the conduction electrons at the $X$ point (in units of $m_{0}$ ) and different band-gap volume deformation potentials $\left(a_{v}^{\alpha}\right)$ (in eV) for ScN.

\begin{tabular}{lccccc}
\hline \hline \multicolumn{1}{c}{ Approach } & $m_{t}^{*}$ & $m_{l}^{*}$ & $a_{v}^{\Gamma-\Gamma}$ & $a_{v}^{\Gamma-X}$ & $a_{v}^{X-X}$ \\
\hline OEPx(cLDA) $-G_{0} W_{0}$ & 0.189 & 1.483 & 1.54 & 2.02 & 2.04 \\
OEPx(cLDA) & 0.253 & 1.450 & 1.07 & 2.06 & 2.21 \\
GGA & 0.139 & 1.625 & 1.43 & 1.87 & 1.92 \\
LDA & 0.126 & 1.570 & 1.36 & 1.95 & 2.03 \\
LDA (Ref. 27) & 0.124 & 1.441 & & & \\
\hline \hline
\end{tabular}

tential. Since the exchange self-energy can be linearly decomposed into a core and a valence contribution no nonlinear core corrections ${ }^{69}$ arise in the Hartree-Fock case and they are expected to be small for OEPx(cLDA) pseudopotentials. ${ }^{39}$ We take the fact that the quasiparticle band structure in the $\mathrm{OEPx}(\mathrm{cLDA})-G_{0} W_{0}$ approach agrees well with (inverse) photoemission data for these materials as an indication that when switching from the local potential in OEPx(cLDA) to the nonlocal self-energy in OEPx(cLDA)$G_{0} W_{0}$ core-valence exchange is treated consistently, as long as OEPx(cLDA) pseudopotentials are used. ${ }^{25}$ Since the semicore $s$ and $p$ states are less localized in $\mathrm{ScN}$ it is computationally feasible to include the entire third shell of Sc as a valence in the pseudopotentials (see Sec. II) and thus to conduct a meaningful comparison between the LDA- $G_{0} W_{0}$ and OEPx(cLDA)- $G_{0} W_{0}$ calculations, which enables us to assess the error bar with respect to a self-consistent $G W$ treatment.

It remains to be added that in previous studies, where a significant starting-point dependence in LDA- $G_{0} W_{0}$ compared to GGA- $G_{0} W_{0}$ calculations was noted, this was either due to structural effects ${ }^{30}$ or significant differences in the ground state ${ }^{31}$ introduced when going from LDA to GGA. Since all calculations in this work were performed at the experimental equilibrium volume the KS band structures in LDA and GGA are very similar (cf. Table II) and LDA- $G_{0} W_{0}$ and GGA- $G_{0} W_{0}$ calculations yield essentially the same result.

Finally, Fig. 1 illustrates that the Sc $3 p$ and Sc $3 s$ bands are significantly lowered by the quasiparticle energy calculations. We have argued recently ${ }^{68}$ that this lowering can to a large degree be attributed to charge-density relaxation effects arising from the removal of an electron from these states. These effects are accounted for in the $G_{0} W_{0}$ approach, but not in DFT Kohn-Sham single-particle energy calculations and are larger for more localized states such as the Sc $3 s$ and $3 p$ bands.

\section{B. Effective masses and deformation potentials}

In this final part we present additional band-structure parameters of $\mathrm{ScN}$, namely, the transverse $\left(m_{t}^{*}\right)$ and longitudinal $\left(m_{l}^{*}\right)$ conduction-band effective masses-at the $X$ pointand the volume band-gap deformation potentials, extracted from our quasiparticle energy calculations. For comparison we will also discuss the corresponding values obtained from LDA, GGA, and OEPx(cLDA).

The conduction-band effective masses at the $X$ point are calculated by fitting a quadratic function to the correspond- ing band-structure energies along the $\Delta(\Gamma-X)$ and $Z(X-W)$ directions for $m_{l}^{*}$ and $m_{t}^{*}$, respectively. A small $k$-point spacing of 0.01 in units of $2 \pi / a$ yields converged effective masses, which are listed in Table III. Our LDA results are in good agreement with those of Ref. 27. As far as an experimental reference is concerned, we are only aware of one study, where a conduction-band effective mass between 0.1 and $0.2 m_{0}$ has been reported. ${ }^{28}$ Apart from the OEPx(cLDA) results, all our KS-DFT and quasiparticle energy calculations give a transverse effective mass in this range, while the longitudinal effective mass is approximately one order of magnitude larger.

To determine the volume deformation potentials $a_{V}^{\alpha}$ (Ref. 70) for the band gaps $E_{g}^{\Gamma-\Gamma}, E_{g}^{\Gamma-X}$, and $E_{g}^{X-X}$, the relevant band gaps are calculated at four different lattice constants around the experimental equilibrium one. For illustration, we show in Fig. 5 the variation of $E_{g}^{\Gamma-\Gamma}$ and $E_{g}^{\Gamma-X}$, with respect to their values at the experimental equilibrium volume, as a function of $\ln \left(V / V_{0}\right)$. The corresponding band gaps are then fitted to a quadratic function of $\ln V$. The linear deformation potentials obtained in this way are listed in Table III. To the best of our knowledge, no experimental or previous theoretical results are available for the deformation potentials of $\mathrm{ScN}$. Based on our results we conclude that $a_{V}^{\alpha}$ is almost insensitive with respect to the computational approaches considered in this article. The only exception is $a_{V}^{\Gamma-\Gamma}$ obtained using the OEPx(cLDA) approach. The fact that the LDA curves in Fig. 5 are very close to the OEPx(cLDA)- $G_{0} W_{0}$ ones, a posteriori justifies the use of the LDA volume-deformation potential for the indirect calculation of the LDA- $G_{0} W_{0}$ band gaps.

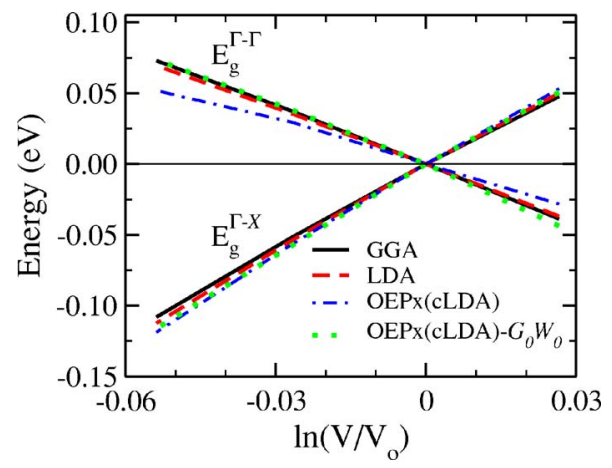

FIG. 5. (Color online) Variation of the indirect band gap $\left(E_{g}^{\Gamma-X}\right)$ and the direct band gap at the $\Gamma$-point $\left(E_{g}^{\Gamma-\Gamma}\right)$ of $\mathrm{ScN}$ with respect to the corresponding band gaps at the experimental equilibrium volume $\left(V_{0}\right)$, as a function of $\ln \left(V / V_{0}\right)$. 


\section{CONCLUSIONS}

Pseudopotential $G_{0} W_{0}$ calculations based on Kohn-Sham density-functional theory calculations in both the LDA and OEPx(cLDA) have been performed for the electronic structure of $\mathrm{ScN}$ in the thermodynamically stable rock-salt phase. To analyze the effects of exchange and correlation the atomic and electronic structures have been studied within DFT for several levels of approximations for the exchange-correlation functional [LDA, GGA and $\operatorname{OEPx}(\mathrm{cLDA})]$. In agreement with previous calculations for $\mathrm{ScN}$, our LDA [OEPx(cLDA)] band gaps are underestimated (overestimated) by about $100 \%$. Despite this large difference, OEPx(cLDA)- $G_{0} W_{0}$ and LDA- $G_{0} W_{0}$ calculations for the quasiparticle band structure agree to within $0.3 \mathrm{eV}$. Our quasiparticle gap of $0.99 \pm 0.15 \mathrm{eV}$ supports the recent observation that $\mathrm{ScN}$ has a much lower indirect band gap than previously thought. The main advantage of the OEPx(cLDA)- $G_{0} W_{0}$ approach lies in the fact that it facilitates a direct calculation of the electronic structure of $\mathrm{ScN}$ at the experimental equilibrium volume, whereas for the LDA- $G_{0} W_{0}$ calculation an indirect approach has to be taken due to the negative LDA band gap.

\section{ACKNOWLEDGMENTS}

We acknowledge stimulating discussions with Arthur Smith, Sixten Boeck, Martin Fuchs, Matthias Wahn, and Christoph Freysoldt. This work was in part supported by the Volkswagen Stiftung/Germany, the DFG research group, nitride-based nanostructures, and the EU's Sixth Framework Programme through the NANOQUANTA (Contract No. NMP4-CT-2004-500198) Network of Excellence.
${ }^{1}$ X. Bai and M. E Kordesch, Appl. Surf. Sci. 175-176, 499 (2001).

${ }^{2}$ D. Gall, I. Petrov, N. Hellgren, L. Hultman, J. E. Sundgren, and J. E. Greene, J. Appl. Phys. 84, 6034 (1998).

${ }^{3}$ J. P. Dismukes, W. M. Yim, and V. S. Ban, J. Cryst. Growth 13/14, 365 (1972).

${ }^{4}$ H. Al-Brithen, H. Yang, and A. Smith, J. Appl. Phys. 96, 3787 (2004).

${ }^{5}$ A. Herwadkar and W. R. L. Lambrecht, Phys. Rev. B 72, 235207 (2005).

${ }^{6}$ N. Farrer and L. Bellaiche, Phys. Rev. B 66, 201203(R) (2002).

${ }^{7}$ V. Ranjan, S. Bin-Omran, L. Bellaiche, and A. Alsaad, Phys. Rev. B 71, 195302 (2005)

${ }^{8}$ V. Ranjan, S. Bin-Omran, D. Sichuga, R. S. Nichols, L. Bellaiche, and A. Alsaad, Phys. Rev. B 72, 085315 (2005).

${ }^{9}$ J. P. Dismukes and T. D. Moustakas, Proc.-Electrochem. Soc. 96-11, 110 (1996).

${ }^{10}$ M. Little and M. E. Kordesch, Appl. Phys. Lett. 78, 2891 (2001).

${ }^{11}$ C. Constantin, H. Al-Brithen, M. B. Haider, D. Ingram, and A. Smith, Phys. Rev. B 70, 193309 (2004).

${ }^{12}$ C. Constantin, M. B. Haider, D. Ingram, A. Smith, N. Sandler, K. Sun, and P. Ordejón, J. Appl. Phys. 98, 123501 (2004).

${ }^{13}$ F. Perjeru, X. Bai, M. I. Ortiz-Lebreros, R. Higgins, and M. E. Kordesch, Appl. Surf. Sci. 175-176, 490 (2001).

${ }^{14}$ V. Ranjan, L. Bellaiche, and E. J. Walter, Phys. Rev. Lett. 90, 257602 (2005).

${ }^{15}$ D. Gall, M. Stadele, K. Jarrendahl, I. Petrov, P. Desjardins, R. T. Haasch, T.-Y. Lee, and J. E. Greene, Phys. Rev. B 63, 125119 (2001).

${ }^{16}$ H. Al-Brithen and A. R. Smith, Appl. Phys. Lett. 77, 2485 (2000).

${ }^{17}$ T. S. Moss, Proc. Phys. Soc. London, Sect. B 67, 775 (1954); L. Burstein, Phys. Rev. 93, 632 (1954).

${ }^{18}$ H. A. Al-Brithen, A. Smith, and D. Gall, Phys. Rev. B 70, 045303 (2004).

${ }^{19}$ R. Monnier, J. Rhyner, T. M. Rice, and D. D. Koelling, Phys. Rev. B 31, 5554 (1985).

${ }^{20}$ A. Neckel, P. Rastl, R. Eibler, P. Weinberger, and K. Schwarz, J. Phys. C 9, 579 (1976).

${ }^{21}$ R. Eibler, M. Dorrer, and A. Neckel, Theor. Chim. Acta 63, 133
(1983).

${ }^{22}$ C. Stampfl, W. Mannstadt, R. Asahi, and A. J. Freeman, Phys. Rev. B 63, 155106 (2001).

${ }^{23}$ L. Hedin, Phys. Rev. 139, A796 (1965).

${ }^{24}$ For a recent review see, W. G. Aulbur, L. Jönsson, and J. W. Wilkins, Solid State Phys. 54, 1 (1999).

${ }^{25}$ P. Rinke, A. Qteish, J. Neugebauer, C. Freysold, and M. Scheffler, New J. Phys. 7, 126 (2005).

${ }^{26}$ G. Onida, L. Reining, and A. Rubio, Rev. Mod. Phys. 74, 601 (2002).

${ }^{27}$ See reference 37 in Ref. 18.

${ }^{28}$ G. Harbeke, E. Meier, and J. P. Dismukes, Opt. Commun. 4, 335 (1972).

${ }^{29}$ P. Rinke, A. Qteish, M. Winkelnkemper, D. Bimberg, J. Neugebauer, and M. Scheffler, Appl. Phys. Lett. 89, 161919 (2006).

${ }^{30}$ J.-L. Li, G.-M. Rignanese, E. K. Chang, X. Blase, and S. G. Louie, Phys. Rev. B 66, 035102 (2002).

${ }^{31}$ J.-L. Li, G.-M. Rignanese, and S. G. Louie, Phys. Rev. B 71, $193102(2005)$

${ }^{32}$ T. Kotani and M. van Schilfgaarde, Solid State Commun. 121, 461 (2002)

${ }^{33}$ M. Usuda, N. Hamada, K. Shiraishi, and A. Oshiyama, Jpn. J. Appl. Phys. 43, L407 (2004).

${ }^{34}$ M. Rohlfing, P. Krüger, and J. Pollmann, Phys. Rev. Lett. 75, 3489 (1995).

${ }^{35}$ M. Rohlfing, P. Krüger, and J. Pollmann, Phys. Rev. B 57, 6485 (1998).

${ }^{36}$ W. Luo, S. Ismail-Beigi, M. L. Cohen, and Steven G. Louie, Phys. Rev. B 66, 195215 (2002).

${ }^{37}$ M. E. Casida, Phys. Rev. A 51, 2005 (1995).

${ }^{38}$ M. Städele, J. A. Majewski, P. Vogl, and A. Görling, Phys. Rev. Lett. 79, 2089 (1997).

${ }^{39}$ M. Städele, M. Moukara, J. A. Majewski, P. Vogl, and A. Görling, Phys. Rev. B 59, 10031 (1999).

${ }^{40}$ W. G. Aulbur, M. Städele, and A. Görling, Phys. Rev. B 62, 7121 (2000).

${ }^{41}$ See, www.sphinxlib.de.

${ }^{42}$ M. Moukara, M. Städele, J. A. Majewski, P. Vogl, and A. Görling, J. Phys.: Condens. Matter 12, 6783 (2000). 
${ }^{43}$ M. Fuchs and M. Scheffler, Comput. Phys. Commun. 119, 67 (1999).

${ }^{44}$ N. Troullier and J. L. Martins, Phys. Rev. B 43, 1993 (1991).

${ }^{45}$ L. Kleinman and D. M. Bylander, Phys. Rev. Lett. 48, 1425 (1982).

${ }^{46}$ C. Stampfl and C. G. Van de Walle, Phys. Rev. B 59, 5521 (1999).

${ }^{47}$ X. Gonze, R. Stumpf, and M. Scheffler, Phys. Rev. B 44, 8503 (1991).

${ }^{48}$ D. M. Ceperley and B. J. Alder, Phys. Rev. Lett. 45, 566 (1980).

${ }^{49}$ J. Perdew and A. Zunger, Phys. Rev. B 23, 5048 (1981).

${ }^{50}$ J. Perdew, K. Burke, and M. Ernzerhof, Phys. Rev. Lett. 77, 3865 (1996).

${ }^{51}$ H. N. Rojas, R. W. Godby, and R. J. Needs, Phys. Rev. Lett. 74, 1827 (1995).

${ }^{52}$ M. M. Rieger, L. Steinbeck, I. White, H. N. Rojas, and R. W. Godby, Comput. Phys. Commun. 117, 211 (1999).

${ }^{53}$ L. Steinbeck, A. Rubio, L. Reining, M. Torrent, I. White, and R. W. Godby, Comput. Phys. Commun. 125, 105 (2000).

${ }^{54}$ C. Freysoldt, P. Eggert, P. Rinke, A. Schindlmayr, R. W. Godby, and M. Scheffler, Comput. Phys. Commun. 176, 1 (2007).

${ }^{55}$ H. J. Monkhorst and J. D. Pack, Phys. Rev. B 13, 5189 (1976).

${ }^{56}$ W. Ku and A. G. Eguiluz, Phys. Rev. Lett. 89, 126401 (2002).

${ }^{57}$ M. L. Tiago, S. Ismail-Beigi, and S. G. Louie, Phys. Rev. B 69, 125212 (2003).

${ }^{58}$ K. Delaney, P. García-González, A. Rubio, P. Rinke, and R. W. Godby, Phys. Rev. Lett. 93, 249701 (2004).

${ }^{59}$ N. E. Zein, S. Y. Savrasov, and G. Kotliar, Phys. Rev. Lett. 96, 226403 (2006).

${ }^{60}$ M. van Schilfgaarde, T. Kotani, and S. Faleev, Phys. Rev. Lett.
96, 226402 (2006).

${ }^{61}$ B. Holm and U. von Barth, Phys. Rev. B 57, 2108 (1998).

${ }^{62}$ M. Marsili, O. Pulci, F. Bechstedt, and R. Del Sole, Phys. Rev. B 72, 115415 (2005).

${ }^{63}$ A. Fleszar and W. Hanke, Phys. Rev. B 71, 045207 (2005).

${ }^{64}$ W. A. Harrison and G. K. Straub, Phys. Rev. B 36, 2695 (1987).

${ }^{65}$ L. Sham and M. Schlüter, Phys. Rev. Lett. 51, 1888 (1983); Phys. Rev. B 32, 3883 (1985).

${ }^{66}$ J. B. Krieger, Y. Li, and G. J. Iafrate, Phys. Rev. B 45, 101 (1992).

${ }^{67}$ M. E. Casida, Phys. Rev. B 59, 4694 (1999).

${ }^{68}$ A. Qteish, A. I. Al-Sharif, M. Fuchs, M. Scheffler, S. Boeck, and J. Neugebauer, Phys. Rev. B 72, 155317 (2005).

${ }^{69}$ S. G. Louie, S. Froyen, and M. L. Cohen, Phys. Rev. B 26, 1738 (1982).

${ }^{70}$ Experimentally, one typically studies the pressure $(P)$ variation of an energy gap, which is usually expressed in terms of the pressure-deformation potential $a_{P}^{\alpha}=\frac{d E_{g}^{\alpha}}{d P}$, where $\alpha$ refers to a certain transition. Using Murnaghan's equation of state, the pressure dependence can be expressed in terms of the equilibrium structural parameters $a_{0}, B_{0}$, and $B_{0}^{\prime}$. Since $B_{0}$ depends sensitively on the exchange-correlation functional (cf. Table I) and the determination of structural parameters in the OEPx(cLDA)$G_{0} W_{0}$ approach is currently not possible, we consider the volume $(V)$ deformation potentials defined as (see, for example, Ref. 72) $a_{V}^{\alpha}=\frac{d E_{g}^{\alpha}}{d \ln V}$, instead. $a_{P}^{\alpha}$ can be obtained from $a_{V}^{\alpha}$ through the relation $a_{P}^{\alpha}=-\left(\frac{1}{B_{0}}\right) a_{V}^{\alpha}$.

${ }^{71}$ N. Takeuchi, Phys. Rev. B 65, 045204 (2002).

${ }^{72}$ S.-H. Wei and A. Zunger, Phys. Rev. B 60, 5404 (1999). 